\title{
Diastereoselective synthesis of tertiary alcohols by nucleophilic addition to $\alpha$-substituted- $\beta$-keto esters
}

\author{
Enrico Marcantoni, ${ }^{a}$ Massimo Massaccesi, ${ }^{\mathrm{a} *}$ Melissa Paoletti, ${ }^{\mathrm{a}}$ and Letizia Sambri ${ }^{\mathrm{b}}$ \\ ${ }^{a}$ Dipartimento di Scienze Chimiche, Università di Camerino, v. S. Agostino 1, 62032 Camerino \\ (MC), Italy \\ ${ }^{b}$ Dipartimento di Chimica Organica “A. Mangini”, Università di Bologna, v.le Risorgimento 4, \\ 40136 Bologna, Italy \\ E-mail: massimo.massaccesi@unicam.it
}

This paper is dedicated to Prof. Giuseppe Bartoli on the occasion of his 65 th birthday

\begin{abstract}
A protocol of nucleophilic addition to $\beta$-keto esters is reported, aimed to the synthesis of tertiary alcohols with high diastereomeric purity. The combined use of organocerium compounds and $\mathrm{TiCl}_{4}$ makes the procedure very efficient and stereoselective with phenyl ketone derivatives. The effect of the nature of the ester function as well as the group bound to the ketone moiety was investigated and rationalized.
\end{abstract}

Keywords: Tertiary alcohols, diastereoselectivity, organocerium, chelate, titanium

\section{Introduction}

The nucleophilic addition of organometallic reagents to carbonyl compounds is a well known reaction, which represents a powerful tool for the construction of carbon-carbon bonds. Particularly attractive is the stereoselective addition to ketones, which produces a tertiary alcohol of defined relative configuration. The attack of Grignard-type reagents to $\beta$-keto ester units is frequently found among the syntheses of complex molecules, for example in the recent preparation of Periplanone $\mathrm{C}^{1}{ }^{1}$ diarylbutylamine pharmacophores ${ }^{2}$ and neodolabellane diterpenoids. ${ }^{3}$ The resulting functionalized alcohols represent valuable building blocks, since the alcoholic function, the carbon framework introduced, and the ester moiety in the $\beta$ - position could be successively manipulated.

We recently reported that organocerium reagents are able to add in highly diastereoselective manner to a metal complex of the starting $\beta$-keto amides, carrying a stereocenter in the $\alpha$ position, to afford $\beta$-hydroxy amides having a tertiary alcoholic fragment. ${ }^{4}$ The high 
nucleophilicity of the organometallic species allows the addition to be carried out at low temperature, thus providing great stereochemical control. ${ }^{5}$ The synthetic strategy adopted for the stereoselective addition of $\mathrm{RMgX}-\mathrm{CeCl}_{3}$ species to $\beta$-keto amides was based on their conversion into the corresponding titanium cyclic complexes. Given that a good variety of carbon frameworks could be introduced by our titanium-mediated protocol, producing in some cases adducts with interesting stereodynamic properties, ${ }^{6}$ the possibility of extending such a procedure to $\alpha$-substituted- $\beta$-keto esters seemed very attractive. The methodology is capable of producing the corresponding $\beta$-hydroxy esters with a stereodefined geometry, which can be seen as precursors of $\beta$-amino acids. ${ }^{7}$ These target molecules might also be obtained by aldol- or Reformatsky condensation; however, the stereochemical outcome of these reactions is hardly controllable. For this reason, the stereoselective addition of organometallic species to $\beta$-keto esters appears the most direct and efficient approach. To the best of our knowledge, only one procedure has been reported where nucleophilic addition of different organometallics ( $\mathrm{Zn}, \mathrm{Al}$, $\mathrm{Mn})$ permits the stereoselective synthesis of $\beta$-hydroxy esters. ${ }^{8}$ The methodology could not be developed into a general solution, and only a limited number of examples of this reaction were reported.

We report here the results of our studies aimed at the elaboration of a general procedure for the synthesis of diastereomerically pure tertiary alcohols by addition of $\mathrm{R}^{2} \mathrm{MgX}-\mathrm{CeCl}_{3}$ to $\beta$-keto esters 1 with an $\alpha$-stereocenter, in the presence of a Lewis acid such as $\mathrm{TiCl}_{4}(\mathrm{Scheme} 1)$.<smiles>[R]OC(=O)C(C)C([R])=O</smiles>

1

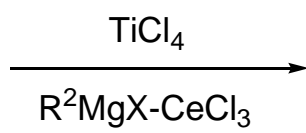

$\mathrm{R}^{2} \mathrm{MgX}-\mathrm{CeCl}_{3}$

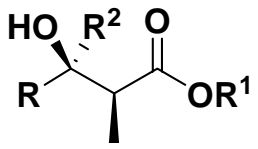

2

\section{Scheme 1}

\section{Results and Discussion}

Chelation-control of stereochemistry is one of the most effective means by which diastereoselective carbonyl addition reactions can be accomplished. ${ }^{9}$ Two decades ago, Molander and co-workers proposed a mechanism based on titanium tetrachloride-initiated cyclization of $\beta$ keto esters in the intramolecular addition of nucleophiles to carbonyl substrates. ${ }^{10}$ Recently, Bartoli and his co-workers demonstrated that the well known ${ }^{11}$ capacity of a Lewis acid such as $\mathrm{TiCl}_{4}$ for forming a stable cyclic arrangement with bidentate compounds promotes a highly stereoselective addition of hydride anions ${ }^{12}$ and organocerium compounds ${ }^{13}$ to $\beta$-functionalized carbonyl compounds. Thus, a high degree of compatibility exists between a titanium(IV) chloride Lewis acid moiety and organocerium reagents, and these two species are able to exert their assigned roles without undesired interactions. These results suggested that we should apply 
such $\mathrm{TiCl}_{4}$ and organocerium reagents' combination to the diastereoselective addition to $\beta$-keto esters.

We chose as model substrates the $\alpha$-methyl- $\beta$-keto esters $\mathbf{1 a}$ and $\mathbf{1 b}$, which were treated with an excess of organocerium derivatives $\mathrm{R}^{2} \mathrm{MgCl}-\mathrm{CeCl}_{3}$ in the presence of a stoichiometric amount of $\mathrm{TiCl}_{4}$ at $-78^{\circ} \mathrm{C}$ (Table 1$)$.

Table 1. Addition of organocerium reagents to $\beta$-keto esters in the presence of $\mathrm{TiCl}_{4}$ at $-78^{\circ} \mathrm{C}$

\begin{tabular}{|c|c|c|c|c|c|c|}
\hline$\beta$-Keto ester & $\mathrm{R}^{2}$ & Solvent & $\begin{array}{l}\text { Starting material } \\
\text { Recovered }(\%)^{\mathrm{a}}\end{array}$ & Product $^{b}$ & Yield $(\%)^{\mathrm{c}}$ & de $(\%)^{d}$ \\
\hline & $\mathrm{Me}$ & $\mathrm{Et}_{2} \mathrm{O}$ & 10 & $2 a a$ & 88 & $>98$ \\
\hline & $\mathrm{PhCH}_{2}$ & THF & 40 & $2 a b$ & 45 & $>98$ \\
\hline & $\mathrm{Ph}$ & $\mathrm{Et}_{2} \mathrm{O}$ & 80 & & $0^{\mathrm{e}}$ & \\
\hline $1 \mathrm{~b}$ & $\mathrm{PhCH}_{2}$ & $\mathrm{THF}$ & 80 & & $0^{\mathrm{e}}$ & \\
\hline
\end{tabular}

${ }^{a}$ After column chromatography; ${ }^{b}$ Products were identified by their NMR and GC/MS spectra; ${ }^{c}$ Yields refer to isolated compounds; ${ }^{\mathrm{d}}$ Determined by ${ }^{1} \mathrm{H}$ - NMR on the crude product; ${ }^{\mathrm{e}}$ Only starting material recovered.

Preliminary investigations revealed that organometallic addition to $\beta$-keto esters was more difficult than the analogous reaction of $\beta$-keto amides. In fact, in the examples studied in Table 1 we recovered part of the starting material, and complete conversion was never reached. It is likely that the low yield of tertiary alcohol was due to enolization of the substrate, even with the use of less basic organocerium reagents. ${ }^{14}$ This is particularly evident with substrate $\mathbf{1 b}$, for which, due to stereoelectronic factors, the addition reaction completely failed, and only starting material was recovered.

The nature of the solvent plays a fundamental role too: in THF the reaction of $\beta$-keto ester 1a, even with a stabilized carbanionic moiety such as a benzyl derivative, resulted in very modest yield of the product 2ab. On the contrary, by the use of the less polar and less coordinating solvent $\mathrm{Et}_{2} \mathrm{O}$, the addition of a carbanion species such as methylcerium derivative afforded the tertiary alcohol $\mathbf{2 a a}$ in $88 \%$ yield. Very likely, the undesired process of enolization of the substrate by the organometallic species becomes predominant when THF was used as a solvent. ${ }^{15}$ However, the diastereoselectivity of the reaction was excellent for both the $\beta$-hydroxy esters obtained, for which only one diastereoisomer was detected by NMR of the crude product. Functionalized tertiary alcohols of defined relative configuration were also successfully 
produced through $\mathrm{TiCl}_{4}$-mediated addition of various organocerium derivatives, using $\mathrm{Et}_{2} \mathrm{O}$ as solvent, to two other $\beta$-keto esters $\mathbf{1 c}$ and 1d, which bear different alkoxy groups. The results are collected in Table 2 .

Table 2. Diastereoselective addition of $\mathrm{R}^{1} \mathrm{MgCl}-\mathrm{CeCl}_{3}$ to $\alpha$-methyl- $\beta$-keto esters.<smiles>[R]OC(=O)C(C)C(=O)c1ccccc1</smiles>

1
1. $\mathrm{TiCl}_{4}, \mathrm{CH}_{2} \mathrm{Cl}_{2},-30^{\circ} \mathrm{C}$

2. $\mathrm{R}^{1} \mathrm{MgCl}-\mathrm{CeCl}_{3}, \mathrm{Et}_{2} \mathrm{O},-78^{\circ} \mathrm{C}$

3. $\mathrm{HCl} \mathrm{aq}$

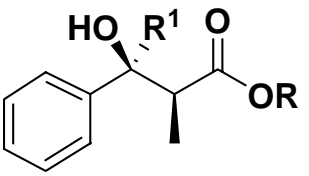

2

\begin{tabular}{|c|c|c|c|c|c|}
\hline Entry & Substrate & $\mathrm{R}^{1}$ in $\mathrm{R}^{1} \mathrm{MgCl}-\mathrm{CeCl}_{3}$ & Product & Yield $(\%)^{\mathrm{a}}$ & $\operatorname{de}(\%)^{b}$ \\
\hline 1 & 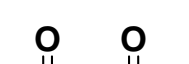 & $\mathrm{Me}$ & $2 \mathbf{a a}$ & 88 & $>98$ \\
\hline 2 & & $\mathrm{PhCH}_{2}$ & $2 \mathbf{a b}$ & 80 & $>98$ \\
\hline 3 & & $t-\mathrm{Bu}$ & $2 a c$ & $46^{\mathrm{c}}$ & $>98$ \\
\hline 4 & $1 a$ & $i-\operatorname{Pr}$ & $2 a d$ & $45^{\mathrm{c}}$ & $>98$ \\
\hline 5 & & $\mathrm{Me}$ & $2 \mathrm{ca}$ & 82 & $>98$ \\
\hline 6 & & $\mathrm{PhCH}_{2}$ & $2 \mathrm{cb}$ & 95 & $>98$ \\
\hline 7 & & $t-\mathrm{Bu}$ & $2 \mathrm{cc}$ & 68 & $>98$ \\
\hline 8 & & $i-\operatorname{Pr}$ & $2 \mathrm{~cd}$ & 60 & $>98$ \\
\hline 9 & $1 c$ & $n-\mathrm{Bu}$ & $2 \mathrm{ce}$ & $45^{\mathrm{c}}$ & $>98$ \\
\hline 10 & & $\mathrm{Et}$ & $2 \mathrm{cf}$ & $45^{\mathrm{c}}$ & $>98$ \\
\hline 11 & & $\mathrm{Me}$ & $2 \mathrm{da}$ & $45^{\mathrm{c}}$ & $>98$ \\
\hline 12 & & $\mathrm{PhCH}_{2}$ & $2 d b$ & 70 & $>98$ \\
\hline 13 & & $t-\mathrm{Bu}$ & $2 \mathrm{dc}$ & $48^{\mathrm{c}}$ & $>98$ \\
\hline 14 & 1d & $n-\mathrm{Bu}$ & $2 \mathrm{de}$ & $35^{\mathrm{c}}$ & $>98$ \\
\hline
\end{tabular}

${ }^{a}$ Yields refer to isolated products; ${ }^{b}$ Determined by ${ }^{1} \mathrm{H}$ - NMR on the crude product; ${ }^{\mathrm{c}}$ More than $30 \%$ of starting material could be recovered after column chromatography.

Our procedure permits the preparation of several tertiary alcohols with nearly complete diastereoselectivity. ${ }^{16}$ Primary, secondary and even tertiary alkyl chains can now be successfully added to the keto moiety. It is worthy of note that even the nucleophilic transfer of bulky groups such as tert-butyl (Table 2, entries 3, 7, 13) and iso-propyl (Table 2, entries 4, 8) proceeds in satisfactory yield. In these particular cases, the highly hindered tertiary alcohols obtained showed 
characteristic stereodynamic effects deriving from restricted rotations, that we described in detail in a previous paper. ${ }^{16}$

None of the by-products that commonly form when tertiary alcohols such as $\mathbf{2}$ are prepared by aldol condensation ${ }^{17}$ are observed in our case: only some starting $\beta$-keto ester is occasionally recovered from the reaction mixture under our conditions. ${ }^{18}$

Our methodology generally proceeds with high diastereoselectivity and with moderate-togood efficiency, and the best results were obtained with $\beta$-keto ester 1c bearing a tert-butoxy group (Table 2, entries 5-10). These experimental evidences show that the formation of a chelation-controlled diastereomer could be reasonably explained by the mechanism depicted in Scheme 2.

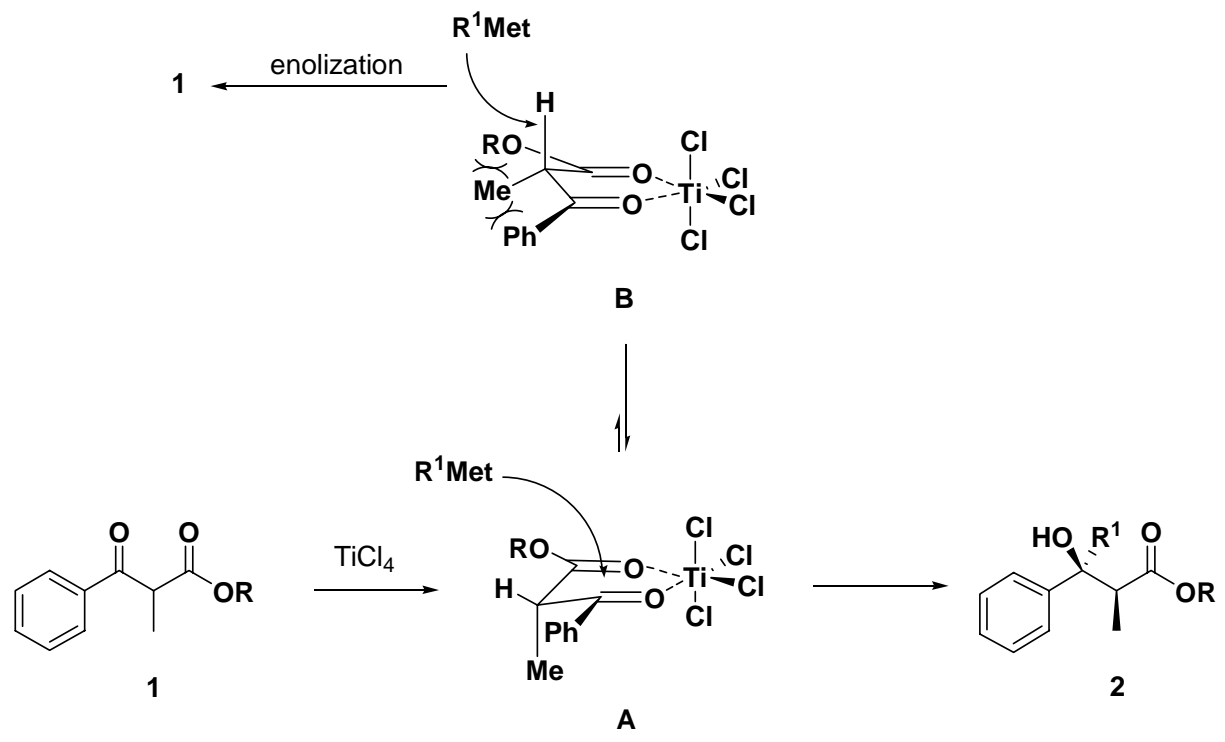

\section{Scheme 2}

The interaction between $\mathrm{TiCl}_{4}$ and the $\beta$-keto ester $\mathbf{1}$, which acts as a bidentate ligand, results in the formation of a rigid cyclic intermediate, whose more stable conformation $\mathbf{A}$ offers a high stereofacial discrimination to the incoming nucleophile $\mathrm{R}^{1}$, affording the $\beta$-hydroxy ester $\mathbf{2}$. The half-chair conformation $\mathbf{B}$, which features destabilizing interactions of the $\alpha$-methyl with both phenyl and alkoxy groups, allows the pseudoaxial proton abstraction ${ }^{19}$ by the basic character of the carbanionic moiety, finally returning the starting ketone 1. It is clear that the reaction will succeed as much as the conformation $\mathbf{B}$ will be disfavored, ${ }^{20}$ and this mostly occurs in the presence of the bulky tert-butyl ester group, as in 1c. The dramatic loss of efficiency of the diastereoselective addition to the $\beta$-keto ester $\mathbf{1 b}$, which bears an ethyl group bound to the ketone, can be understood as a consequence of the lack of the afore-mentioned conformational bias. ${ }^{21}$ Thus, an important remark is that the efficiency of our methodology depends on the nature of the carbonyl- substituent in the $\beta$-keto ester substrate. Furthermore in contrast with the Lewis acid mediated reduction of $\beta$-oxygenated ketones, where a lower diastereoselectivity was 
observed with an alkyl carbonyl moiety than with a phenyl carbonyl moiety, ${ }^{22}$ in our nucleophilic addition to $\beta$-keto esters such as $\mathbf{1 b}$ the recovery of the starting material is the predominant effect (See Table 1).

In conclusion, a Lewis acid moiety can be easily introduced onto a $\beta$-keto ester possessing an $\alpha$-stereocenter, and the system assumes a stable cyclic arrangement which offers great stereofacial discrimination. During our study in developing new methods for stereoselective addition of nucleophiles to $\alpha$-substituted- $\beta$-keto esters, we have established an efficient protocol using an organocerium/ $/ \mathrm{TiCl}_{4}$ combination. A large variety of carbon frameworks can be introduced with good efficiency into phenyl carbonyl moieties, and uncommon tertiary alcohols functionalized at the $\beta$ - position were obtained. The absolute diastereoselectivity of the reaction undoubtedly makes such a protocol a useful tool in organic synthesis, and we are currently working towards expanding the scope of $\mathrm{RMgX}-\mathrm{CeCl}_{3} / \mathrm{TiCl}_{4}$ system to the modern synthetic methodologies.

\section{Experimental Section}

General Procedures. Dry $\mathrm{Et}_{2} \mathrm{O}$ was distilled over $\mathrm{Na}$ /benzophenone before use. Grignard reagents were purchased as solutions in $\mathrm{Et}_{2} \mathrm{O}$ and titrated before use. All reactions were performed under Argon atmosphere. Mass spectra were recorded on a GC-HP 5980 instrument, fitted with a HP 5971 mass detector. Flash column chromatography was carried out using Merck Silica gel $60(0.040-0.063 \mathrm{~mm})$.

The $\beta$-keto ester 1 a was prepared following a reported procedure, ${ }^{23}$ by condensation of ethyl propanoate with ethyl benzoate; $\mathbf{1 b}^{24}$ and $\mathbf{1} \mathbf{c}^{25}$ were prepared according to reported procedures. Spectroscopic data of $\mathbf{1 a},{ }^{26} \mathbf{1 b}^{27}$ and $\mathbf{1} \mathbf{c}^{24}$ are in agreement with those reported in the literature.

Phenyl 2-methyl-3-oxo-3-phenylpropanoate (1d). $\mathrm{TiCl}_{4}\left(1.2 \mathrm{mmol}, 1 \mathrm{M}\right.$ solution in $\left.\mathrm{CH}_{2} \mathrm{Cl}_{2}\right)$ and $\mathrm{Et}_{3} \mathrm{~N}(1.4 \mathrm{mmol})$ were successively added to a stirred solution of phenyl propanoate ${ }^{28}$ (1 mmol) in $5 \mathrm{ml}$ of dry $\mathrm{CH}_{2} \mathrm{Cl}_{2}$ at $-78^{\circ} \mathrm{C}$. After stirring at the same temperature for $30 \mathrm{~min}$, a solution of ethyl benzoate $(1.2 \mathrm{mmol})$ in $\mathrm{CH}_{2} \mathrm{Cl}_{2}$ was added to the mixture, following by stirring at $-78^{\circ} \mathrm{C}$ for $4 \mathrm{~h}$. The reaction was quenched with aqueous $\mathrm{HCl}(1 \mathrm{M})$, then extracted with $\mathrm{CH}_{2} \mathrm{Cl}_{2}$. The crude oil was purified by silica gel column chromatography (light petroleum:Et $\mathrm{O}_{2}$ 9:1), to afford the product in 70\% yield; ${ }^{1} \mathrm{H}-\mathrm{NMR}\left(300 \mathrm{MHz}, \mathrm{CDCl}_{3}\right) \delta 1.63\left(\mathrm{~d}, 3 \mathrm{H}, \mathrm{CH}_{3}\right.$, $\left.J_{H H}=7.0 \mathrm{~Hz}\right), 4.61\left(\mathrm{q}, 1 \mathrm{H}, \mathrm{CH}, J_{H H}=7.0 \mathrm{~Hz}\right), 6.95-7.05(\mathrm{~m}, 2 \mathrm{H}, \mathrm{Ph}), 7.15-7.35(\mathrm{~m}, 3 \mathrm{H}, \mathrm{Ph}), 7.50-$ 7.65 (m, 3H, Ph), 8.00-8.10 (m, 2H, Ph); ${ }^{13} \mathrm{C}-\mathrm{NMR}\left(75 \mathrm{MHz}, \mathrm{CDCl}_{3}\right) \delta 13.8\left(\mathrm{CH}_{3}\right), 48.4(\mathrm{CH})$, $121.2(\mathrm{CH}), 125.9(\mathrm{CH}), 128.5(\mathrm{CH}), 128.8(\mathrm{CH}), 129.3(\mathrm{CH}), 133.6(\mathrm{CH}), 135.5(\mathrm{C}), 150.5(\mathrm{C})$, 169.5 (C), 195.6 (C); EI-MS m/z 161, 105 (100), 94, 77. 
Diastereoselective addition of organocerium reagents to $\boldsymbol{\beta}$-keto esters.

General procedure. To a solution of $\beta$-keto ester $1(1 \mathrm{mmol})$ in dry $\mathrm{CH}_{2} \mathrm{Cl}_{2}(10 \mathrm{~mL}), \mathrm{TiCl}_{4}(1.2$ mmol, a $1 \mathrm{M}$ solution in $\mathrm{CH}_{2} \mathrm{Cl}_{2}$ ) was added at $-30^{\circ} \mathrm{C}$. After 30 min the reaction was cooled at $78^{\circ} \mathrm{C}$, and the separately prepared ${ }^{29}$ solution of organocerium reagent $(6 \mathrm{mmol})$ in $\mathrm{Et}_{2} \mathrm{O}$ was introduced. The reaction was stirred at this temperature for $1 \mathrm{~h}$, then quenched with aqueous $\mathrm{HCl}$ $(1 \mathrm{M})$. The product was extracted with $\mathrm{Et}_{2} \mathrm{O}$, and the combined organic layers were dried over $\mathrm{MgSO}_{4}$ and concentrated. The crude product was purified by silica gel column chromatography with petroleum ether $/ \mathrm{Et}_{2} \mathrm{O}$ as eluent.

\section{Compound characterization}

The analytical data of the products $2 \mathbf{a a},{ }^{30} \mathbf{2 a c},{ }^{16} \mathbf{2 a d},{ }^{16} \mathbf{2} \mathbf{c c},{ }^{16} \mathbf{2} \mathbf{d c}{ }^{16}$ have already been reported in the literature.

(2S*, 3S*)-Ethyl 3-hydroxy-2-methyl-3,4-diphenylbutanoate (2ab). ${ }^{1} \mathrm{H}$ NMR (300 MHz, $\left.\mathrm{CDCl}_{3}\right) \delta 0.96\left(\mathrm{~d}, 3 \mathrm{H}, \mathrm{CH}_{3}, J_{\mathrm{HH}}=7.1 \mathrm{~Hz}\right), 1.30\left(\mathrm{t}, 3 \mathrm{H}, \mathrm{CH}_{3}, J_{H H}=7.1 \mathrm{~Hz}\right), 2.95-3.15\left(\mathrm{~m}, 3 \mathrm{H}, \mathrm{CH}_{2}\right.$ and $\mathrm{CH}), 4.15-4.25\left(\mathrm{~m}, 3 \mathrm{H}, \mathrm{CH}_{2} \mathrm{O}\right.$ e $\left.\mathrm{OH}\right), 6.80-7.25(\mathrm{~m}, 10 \mathrm{H}, \mathrm{Ph}) ;{ }^{13} \mathrm{C} \mathrm{NMR}\left(75 \mathrm{MHz}, \mathrm{CDCl}_{3}\right) \delta$ $13.1\left(\mathrm{CH}_{3}\right), 14.1\left(\mathrm{CH}_{3}\right), 47.5(\mathrm{CH}), 48.5\left(\mathrm{CH}_{2}\right), 60.9\left(\mathrm{CH}_{2}\right), 77.3(\mathrm{C}), 125.6(\mathrm{CH}), 126.1(\mathrm{CH})$, $126.5(\mathrm{CH}), 127.4(\mathrm{CH}), 127.7(\mathrm{CH}), 130.5(\mathrm{CH}), 136.3(\mathrm{C}), 142.5(\mathrm{C}), 177.5(\mathrm{C}) ; \mathrm{EI}-\mathrm{MS} \mathrm{m} / \mathrm{z}$ 207, 161, 105 (100), 91, 77.

(2S*,3S*)-tert-Butyl 3-hydroxy-2-methyl -3-phenylbutanoate (2ca). ${ }^{1} \mathrm{H}$ NMR (300 MHz, $\left.\mathrm{CDCl}_{3}\right) \delta 0.92\left(\mathrm{~d}, 3 \mathrm{H}, \mathrm{CH}_{3}, J_{\mathrm{HH}}=7.1 \mathrm{~Hz}\right), 1.50\left(\mathrm{~s}, 9 \mathrm{H},\left(\mathrm{CH}_{3}\right)_{3} \mathrm{C}\right) 1.57\left(\mathrm{~s}, 3 \mathrm{H}, \mathrm{CH}_{3}\right), 2.71(\mathrm{q}, 1 \mathrm{H}$, $\left.\mathrm{CH}, J_{\mathrm{HH}}=7.1 \mathrm{~Hz}\right), 4.14(\mathrm{bs}, 1 \mathrm{H}, \mathrm{OH}), 7.20-7.45(\mathrm{~m}, 5 \mathrm{H}, \mathrm{Ph}) ;{ }^{13} \mathrm{C} \mathrm{NMR}\left(75 \mathrm{MHz}, \mathrm{CDCl}_{3}\right) \delta 12.7$ $\left(\mathrm{CH}_{3}\right), 28.0\left(\mathrm{CH}_{3}\right), 29.9\left(\mathrm{CH}_{3}\right), 49.9(\mathrm{CH}), 74.3(\mathrm{C}), 81.7(\mathrm{C}), 124.8(\mathrm{CH}), 126.5(\mathrm{CH}), 128.0$ (CH), $145.3(\mathrm{C}), 176.8(\mathrm{C})$.

(2S*,3S*)-tert-Butyl 3-hydroxy-2-methyl-3,4-diphenylbutanoate (2cb). ${ }^{1} \mathrm{H}$ NMR (300 MHz, $\left.\mathrm{CDCl}_{3}\right) \delta 0.97\left(\mathrm{~d}, 3 \mathrm{H}, \mathrm{CH}_{3}, J_{H H}=7.1 \mathrm{~Hz}\right), 1.55\left(\mathrm{~s}, 9 \mathrm{H},\left(\mathrm{CH}_{3}\right)_{3} \mathrm{C}\right), 2.95$ (q, 1H, CH, $J_{H H}=7.1 \mathrm{~Hz}$ ), 3.11 (AB system, 2H, $\left.\mathrm{CH}_{2}, J_{H H}=13.3 \mathrm{~Hz}\right), 4.38(\mathrm{~s}, 1 \mathrm{H}, \mathrm{OH}), 6.80-6.85(\mathrm{~m}, 2 \mathrm{H}, \mathrm{Ph}), 7.05-7.25$ $(\mathrm{m}, 8 \mathrm{H}, \mathrm{Ph}) ;{ }^{13} \mathrm{C}$ NMR $\left(75 \mathrm{MHz}, \mathrm{CDCl}_{3}\right) \delta 12.9\left(\mathrm{CH}_{3}\right), 28.1\left(\mathrm{CH}_{3}\right), 48.3\left(\mathrm{CH}_{2}\right), 48.5(\mathrm{CH}), 77.5$ $(\mathrm{C}), 82.0(\mathrm{C}), 125.8(\mathrm{CH}), 126.0(\mathrm{CH}), 126.4(\mathrm{CH}), 127.4(\mathrm{CH}), 127.7(\mathrm{CH}), 130.6(\mathrm{CH}), 136.7$ (C), 142.5 (C), 177.2 (C); EI-MS m/z 281, 253, 207, 197, 179, 161, 105 (100), 91, 77, 56.

(2S*,3S*)-tert-Butyl 3-hydroxy-2,4-dimethyl-3-phenylpentanoate (2cd). ${ }^{1} \mathrm{H}$ NMR (300 MHz, $\left.\mathrm{CDCl}_{3}\right) \delta 0.77\left(\mathrm{~d}, 3 \mathrm{H}, \mathrm{CH}_{3}, J_{\mathrm{HH}}=6.8 \mathrm{~Hz}\right), 0.87$ (d, 3H, $\left.\mathrm{CH}_{3}, J_{\mathrm{HH}}=6.8 \mathrm{~Hz}\right), 0.98\left(\mathrm{~d}, 3 \mathrm{H}, \mathrm{CH}_{3}\right.$, $\left.J_{H H}=7.1 \mathrm{~Hz}\right), 1.48\left(\mathrm{~s}, 9 \mathrm{H},\left(\mathrm{CH}_{3}\right)_{3} \mathrm{C}\right), 2.02$ (heptet, $\left.1 \mathrm{H}, J_{H H}=6.8 \mathrm{~Hz}\right), 3.13\left(\mathrm{q}, 1 \mathrm{H}, \mathrm{CH}, J_{H H}=7.1\right.$ $\mathrm{Hz}), 4.13$ (s, 1H, OH), 7.20-7.40 (m, 5H, Ph); ); ${ }^{13} \mathrm{C}$ NMR (75 MHz, $\left.\mathrm{CDCl}_{3}\right) \delta 13.3\left(\mathrm{CH}_{3}\right), 17.3$ $\left(\mathrm{CH}_{3}\right), 17.9\left(\mathrm{CH}_{3}\right), 27.9\left(\mathrm{CH}_{3}\right), 37.8(\mathrm{CH}), 45.7(\mathrm{CH}), 79.4(\mathrm{C}), 81.6(\mathrm{C}), 126.3(\mathrm{CH}), 126.4$ (CH), $127.4(\mathrm{CH}), 140.9$ (C), 176.9 (C); EI-MS m/z 235, 205, 179, 161, 149, 105 (100), 77, 57.

(2S*,3S*)-tert-Butyl 3-hydroxy-2-methyl-3-phenylheptanoate (2ce). ${ }^{1} \mathrm{H}$ NMR (300 MHz, $\left.\mathrm{CDCl}_{3}\right) \delta 0.78\left(\mathrm{t}, 3 \mathrm{H}, \mathrm{CH}_{3}, J_{H H}=7.3 \mathrm{~Hz}\right), 0.89\left(\mathrm{~d}, 3 \mathrm{H}, \mathrm{CH}_{3}, J_{H H}=7.1 \mathrm{~Hz}\right), 1.15-1.40(\mathrm{~m}, 4 \mathrm{H}$, $\left.2 \mathrm{CH}_{2}\right), 1.51\left(\mathrm{~s}, 9 \mathrm{H},\left(\mathrm{CH}_{3}\right)_{3} \mathrm{C}\right), 1.72\left(\mathrm{dt}, 1 \mathrm{H}, \mathrm{CH}_{2}, J_{H H}=12.2 \mathrm{~Hz}, J_{H H}=4.5 \mathrm{~Hz}\right), 1.87\left(\mathrm{dt}, 1 \mathrm{H}, \mathrm{CH}_{2}\right.$, $\left.J_{H H}=12.2 \mathrm{~Hz}, J_{H H}=4.5 \mathrm{~Hz}\right), 2.71\left(\mathrm{q}, 1 \mathrm{H}, \mathrm{CH}, J_{H H}=7.1 \mathrm{~Hz}\right), 3.97$ (bs, 1H, OH), 7.15-7-40 (m, 5H, $\mathrm{Ph}) ;{ }^{13} \mathrm{C}$ NMR $\left(75 \mathrm{MHz}, \mathrm{CDCl}_{3}\right) \delta 12.6\left(\mathrm{CH}_{3}\right), 13.9\left(\mathrm{CH}_{3}\right), 22.9\left(\mathrm{CH}_{2}\right), 25.6\left(\mathrm{CH}_{2}\right), 28.0\left(\mathrm{CH}_{3}\right)$, 
$41.7\left(\mathrm{CH}_{2}\right), 49.6(\mathrm{CH}), 76.9(\mathrm{C}), 81.7(\mathrm{C}), 125.5(\mathrm{CH}), 126.3(\mathrm{CH}), 127.9(\mathrm{CH}), 143.3(\mathrm{C}), 177.1$ (C); EI-MS m/z 235, 219, 179, 163, 120, 105 (100), 91, 77, 57.

(2S*,3S*)-tert-Butyl 3-hydroxy-2-methyl-3-phenylpentanoate (2cf). ${ }^{1} \mathrm{H}$ NMR (300 $\mathrm{MHz}$, $\left.\mathrm{CDCl}_{3}\right) \delta 0.65\left(\mathrm{t}, 3 \mathrm{H}, \mathrm{CH}_{3}, J_{\mathrm{HH}}=7.3 \mathrm{~Hz}\right), 0.89\left(\mathrm{~d}, 3 \mathrm{H}, \mathrm{CH}_{3}, J_{\mathrm{HH}}=7.2 \mathrm{~Hz}\right), 1.49\left(\mathrm{~s}, 9 \mathrm{H},\left(\mathrm{CH}_{3}\right)_{3} \mathrm{C}\right)$, $1.83\left(\mathrm{~m}, 2 \mathrm{H}, \mathrm{CH}_{2}\right), 2.71$ (q, $1 \mathrm{H}, \mathrm{CH}, J_{H H}=7.2 \mathrm{~Hz}$ ), 3.90 (bs, $\left.1 \mathrm{H}, \mathrm{OH}\right), 7.15-7.40(\mathrm{~m}, 5 \mathrm{H}, \mathrm{Ph}) ;{ }^{13} \mathrm{C}$ NMR $\left(75 \mathrm{MHz}, \mathrm{CDCl}_{3}\right) \delta 7.9\left(\mathrm{CH}_{3}\right), 12.7\left(\mathrm{CH}_{3}\right), 28.0\left(\mathrm{CH}_{3}\right), 34.4\left(\mathrm{CH}_{2}\right), 49.4(\mathrm{CH}), 77.3(\mathrm{C})$, $81.6(\mathrm{C}), 125.5(\mathrm{CH}), 126.3(\mathrm{CH}), 127.8(\mathrm{CH}), 142.8(\mathrm{C}), 177.2(\mathrm{C})$; EI-MS m/z 235, 191, 179, 161, 135, 105 (100), 77, 57.

(2S*,3S*)-Phenyl 3-hydroxy-2-methyl-3,4-diphenylbutanoate (2db). ${ }^{1} \mathrm{H}$ NMR (300 MHz, $\left.\mathrm{CDCl}_{3}\right) \delta 1.09\left(\mathrm{~d}, 3 \mathrm{H}, \mathrm{CH}_{3}, J_{\mathrm{HH}}=7.1 \mathrm{~Hz}\right), 3.10-3.28\left(\mathrm{~m}, 3 \mathrm{H}, \mathrm{CH}_{2}\right.$ e $\left.\mathrm{CH}\right), 3.78(\mathrm{bs}, 1 \mathrm{H}, \mathrm{OH}), 6.70-$ $6.80(\mathrm{~m}, 2 \mathrm{H}, \mathrm{Ph}), 7.00-7.35(\mathrm{~m}, 13 \mathrm{H}, \mathrm{Ph}) ;{ }^{13} \mathrm{C} \mathrm{NMR}\left(75 \mathrm{MHz}, \mathrm{CDCl}_{3}\right) \delta 13.1\left(\mathrm{CH}_{3}\right), 47.9\left(\mathrm{CH}_{2}\right)$, $48.3(\mathrm{CH}), 77.5(\mathrm{C}), 121.3(\mathrm{CH}), 125.7(\mathrm{CH}), 126.2(\mathrm{CH}), 126.3(\mathrm{CH}), 126.7(\mathrm{CH}), 127.5(\mathrm{CH})$, $127.8(\mathrm{CH}), 129.5(\mathrm{CH}), 130.5(\mathrm{CH}), 136.1(\mathrm{C}), 142.1(\mathrm{C}), 150.2(\mathrm{C}), 175.9(\mathrm{C})$.

(2S*,3S*)-Phenyl 3-hydroxy-2-methyl-3-phenylheptanoate (2de). ${ }^{1} \mathrm{H}$ NMR (300 $\mathrm{MHz}$, $\left.\mathrm{CDCl}_{3}\right) \delta 0.80\left(\mathrm{t}, 3 \mathrm{H}, \mathrm{CH}_{3}, J_{\mathrm{HH}}=7.2 \mathrm{~Hz}\right), 0.85-1.00\left(\mathrm{~m}, 1 \mathrm{H}, \mathrm{CH}_{2}\right) 1.09\left(\mathrm{~d}, 3 \mathrm{H}, \mathrm{CH}_{3}, J_{\mathrm{HH}}=7.1 \mathrm{~Hz}\right)$, 1.20-1.45 (m, 3H, 2CH $), 1.86\left(\mathrm{dt}, 1 \mathrm{H}, \mathrm{CH}_{2}, J_{H H}=12.3 \mathrm{~Hz}, J_{H H}=4.8 \mathrm{~Hz}\right), 2.04$ (dt, $1 \mathrm{H}, \mathrm{CH}_{2}$, $\left.J_{H H}=12.3 \mathrm{~Hz}, J_{H H}=4.8 \mathrm{~Hz}\right), 3.10\left(\mathrm{q}, 1 \mathrm{H}, \mathrm{CH}, J_{H H}=7.1 \mathrm{~Hz}\right), 3.50(\mathrm{bs}, 1 \mathrm{H}, \mathrm{OH}), 7.09$ (d, 2H Ph, $\left.J_{H H}=8.1 \mathrm{~Hz}\right), 7.25-7.45(\mathrm{~m}, 8 \mathrm{H}, \mathrm{Ph}) ; 13 \mathrm{C} \mathrm{NMR}\left(75 \mathrm{MHz}, \mathrm{CDCl}_{3}\right) \delta 12.7\left(\mathrm{CH}_{3}\right), 13.9\left(\mathrm{CH}_{3}\right), 22.9$ $\left(\mathrm{CH}_{2}\right), 25.6\left(\mathrm{CH}_{2}\right), 41.6\left(\mathrm{CH}_{2}\right), 49.3(\mathrm{CH}), 77.1(\mathrm{C}), 121.4(\mathrm{CH}), 125.5(\mathrm{CH}), 126.2(\mathrm{CH}), 126.6$ $(\mathrm{CH}), 128.1(\mathrm{CH}), 129.5(\mathrm{CH}), 142.7(\mathrm{C}), 150.2(\mathrm{C}), 176.1(\mathrm{C})$.

\section{Acknowledgments}

This work was carried out in the framework of the National Project "Studio degli Aspetti Teorici ed Applicativi degli Aggregati di Molecole Target su Siti Catalitici Stereoselettivi" supported by MIUR, Rome, and by University of Camerino. M. P. gratefully acknowledges Pfizer-Ascoli Piceno Plant for PhD Scholarship.

\section{References}

1. Ivkovic, A.; Matovic, R.; Saicic, R. N. Org. Lett. 2004, 6, 1221.

2. Maertens, F.; Toppet, S.; Compernolle, F.; Hoornaert, G. J. Eur. J. Org. Chem. 2004, 2707.

3. Mehta, G.; Umarye, J. D. Tetrahedron Lett. 2003, 44, 7285.

4. (1) Bartoli, G.; Bosco, M.; Marcantoni, E.; Massaccesi, M.; Rinaldi, S.; Sambri, L. Tetrahedron Lett. 2001, 42, 6093. (2) Bartoli, G.; Bosco, M.; Marcantoni, E.; Massaccesi, M.; Rinaldi, S.; Sambri, L. Synthesis 2004, 18, 3092. 
5. (1) Fürstner, A; Weintritt, H. J. Am. Chem. Soc. 1998, 120, 2817. (2) Enders, D.; Meiers, M. Angew. Chem. Int. Ed. 1996, 36, 2261. (3) Denmark, S. E.; Weber, T.; Piotrowski, D. W. J. Am. Chem. Soc. 1987, 109, 2224.

6. Bartoli, G.; Grilli, S.; Lunazzi, L.; Massaccesi, M.; Mazzanti, A.; Rinaldi, S. J. Org. Chem. 2002, 67, 2659.

7. Carda, M.; Murga, J.; Falomir, E.; Gonzàlez, F.; Marco, J. A. Tetrahedron: Asymmetry 2000, 11, 3211.

8. Taniguchi, M.; Fujii, H.; Oshima, K.; Utimoto, K. Bull. Chem. Soc. Jpn. 1994, 67, 2514.

9. Eliel, E. L.; Wilen, S. H.; Mander, L. N. Stereochemistry of Organic Compounds; Wiley Press: New York, 1994; Chap. 12, pp 835-971.

10. Molander, G. A.; Andrews, S. W. Tetrahedron Lett. 1986, 27, 3115.

11. Reetz, M. T. Acc. Chem. Res. 1993, 26, 462.

12. (1) Bartoli, G.; Bartolacci, M.; Cortese, M.; Marcantoni, E.; Massaccesi, M. Sambri, L. Eur. J. Org. Chem. 2004, 2359. (2) Bartoli, G.; Bosco, M.; Marcantoni, E.; Massaccesi, M.; Rinaldi, S.; Sambri, L. Eur. J. Org. Chem. 2001, 4679. (3) Marcantoni, E.; Alessandrini, M.; Malavolta, M.; Bartoli, G.; Bellucci, M. C.; Sambri, L.; Dalpozzo, R. J. Org. Chem. 1999, 64, 1986. (4) Bartoli, G.; Bosco, M.; Dalpozzo, R.; Marcantoni, E.; Sambri, L. Chem. Eur. J. 1997, 3, 1941.

13. (1) Bartoli, G.; Bosco, M.; Di Martino, E.; Marcantoni, E.; Sambri, L. Eur. J. Org. Chem. 2001, 2901. (2) Bartoli, G.; Bellucci, M. C.; Bosco, M.; Marcantoni, E.; Sambri, L. Chem. Eur. J. 1998, 4, 2154.

14. Organocerium compounds are known to have less basic and more nucleophilic character with respect to analogous Grignard reagents. For recent papers about their features and applications, see: (1) Conlon, D. A.; Kumke, D.; Moeder, C.; Hardiman, M.; Hutson, G.; Sailer, L. Adv. Synth. Catal. 2004, 346, 1307. (2) Liu, H.; Shia, K.; Shang, X.; Zhu, B. Tetrahedron 1999, 55, 3803.

15. The same solvent effect was observed in the reaction with $\beta$-keto amides, especially in the case of addition of non-stabilized carbanionic moieties (Ref. 4).

16. The relative configuration of the products was assigned by NOE experiments: Bartoli, G.; Lunazzi, L.; Massaccesi, M.; Mazzanti, A. J. Org. Chem. 2004, 69, 821.

17. Molander, G. A.; Etter, J. B.; Zinke, P. W. J. Am. Chem. Soc. 1987, 109, 453.

18. The starting materials can be easily separated from the $\beta$-hydroxy esters by column chromatography.

19. Bartoli, G.; Marcantoni, E.; Petrini, M. Angew. Chem. Int. Ed. 1993, 32, 1061.

20. A further factor to be considered is the grade of complexation of the $\beta$-keto ester with the Lewis Acid. From preliminary NMR investigations, the spectrum recorded at $-30^{\circ} \mathrm{C}$ after mixing $\mathbf{1 b}$ with $\mathrm{TiCl}_{4}$ in $\mathrm{CD}_{2} \mathrm{Cl}_{2}$ showed the presence of two species, likely corresponding to the uncoordinated substrate and the hypothesized intermediate complex. Undoubtedly, the deprotonation on the uncomplexed dicarbonyl compound would take place very easily, to afford the stabilized enolate species. 
21. Sarko, C. R.; Guch, I. C.; DiMare, M. J. Org. Chem. 1994, 59, 705.

22. (1) Sarko, C. R.; Collibee, S. E.; Knorr, A. L.; DiMare, M. J. Org. Chem. 1996, 61, 868. (2) Faucher, A. M.; Brochu, C.; Landry, S. R.; Duchesne, I.; Hantos, S.; Roy, A.; Myles, A.; Legault, C. Tetrahedron Lett. 1998, 39, 8425.

23. Bartoli, G.; Bosco, M.; Marcantoni, E.; Sambri, L. Tetrahedron Lett. 1996, 37, 7421.

24. Newman M.S.; Evans, F. J. J. Am. Chem. Soc. 1955, 77, 946.

25. Turner, J. A.; Jacks, W. S. J. Org. Chem. 1989, 54, 4229.

26. Katayama, S.; Fukuda, K.; Watanabe, T.; Yamauchi, M. Synthesis 1988, 178.

27. Bellus, D.; Martin, P.; Sauter, H.; Winkler, T. Helv. Chim. Acta 1980, 63, 1130.

28. Prepared according to: Bartoli, G.; Bosco, M.; Dalpozzo, R.; Marcantoni, E.; Massaccesi, M.; Sambri, L. Eur. J. Org. Chem. 2003, 4611.

29. Imamoto, T.; Takeda, N. Org. Synth. 1998, 76, 228.

30. Ross, N. A.; Bartsch, R. A. J. Org. Chem. 2003, 68, 360. 